\title{
Muscle belly in the tunnel: an unusual cause of carpal tunnel syndrome in a patient with spinal cord injury
}

\author{
Esra Giray $\mathbb{D}^{1} \cdot$ Kardelen Gencer Atalay ${ }^{1} \cdot$ Sefa Kurt ${ }^{1} \cdot$ IIlker Yağcı ${ }^{1}$
}

Received: 3 March 2019 / Revised: 13 May 2019 / Accepted: 20 May 2019

(c) International Spinal Cord Society 2019

\begin{abstract}
Introduction Carpal tunnel syndrome, entrapment of median nerve at the wrist, is one of the most commonly encountered peripheral neuropathies in the upper extremity. It is also common in individuals with spinal cord injury due to repetitive movements during wheelchair use. Although it is well known that prevalence of carpal tunnel syndrome is high in individuals with spinal cord injury, no previous study identified aberrant muscle as the cause.

Case presentation A 43-year-old man with T10 incomplete, ASIA Impairment Scale (AIS) C, patient with paraplegia who is a wheelchair basketball player presented to our electrodiagnostic laboratory with complaints of bilateral hand numbness after intensive training for a local veteran wheelchair basketball tournament. Nerve conduction studies showed carpal tunnel syndrome. Ultrasonographic assessment of carpal tunnel revealed the presence of abnormal muscle in the carpal tunnel encroaching the median nerve dynamically.

Discussion It is important to identify the underlying cause of carpal tunnel syndrome, which is a common cause of upper extremity impairment in individuals with spinal cord injury because individuals with spinal cord injury who use wheelchair depend on their arms for mobility, transfers, and most activities of daily life.
\end{abstract}

\section{Introduction}

Carpal tunnel syndrome is one of the most commonly encountered peripheral neuropathies in the upper extremity; it is defined as an entrapment syndrome of the median nerve at the wrist [1]. While most cases are idiopathic, several extrinsic or intrinsic processes are responsible for the etiology of carpal tunnel syndrome; some of these processes are common while others are rare [2,3]. While the carpal tunnel is thought to only contain tendons, ultrasound imaging shows that it might contain echogenic muscle bellies in addition to other components of the carpal tunnel. These aberrant structures most often appear as elongated,

Supplementary information The online version of this article (https:// doi.org/10.1038/s41394-019-0201-0) contains supplementary material, which is available to authorized users.

Esra Giray

girayesra@hotmail.com

esra.giray@marmara.edu.tr

1 Department of Physical Medicine and Rehabilitation, Marmara University School of Medicine, İstanbul, Turkey hypertrophic normal muscle bellies, which intrude into tunnel [4]. There is a lack of literature describing the ultrasonographic occurrence of aberrant muscle bellies in carpal tunnel, while other anatomical variations, such as bifurcated median nerves and persistent median arteries, have been previously reported several times [4]. In patients with an aberrant muscle belly in the carpal tunnel, the tunnel becomes more crowded, which can lead to entrapment of the median nerve. Few studies have reported on the abnormal appearance of the palmaris longus, the flexor digitorum superficialis, and the lumbrical muscles, or aberrant muscles, such as the palmaris profundus and the accessory palmaris longus. Hypertrophy of these muscles that cause carpal tunnel syndrome have been demonstrated in manual laborers [2]. In this paper, we present a case of carpal tunnel syndrome due to hypertrophy of the flexor digitorum superficialis of index finger in a patient with spinal cord injury who is a wheelchair basketball player.

\section{Case presentation}

A 43-year-old man with T10 incomplete, ASIA Impairment Scale (AIS) C, patient with paraplegia who is a wheelchair 


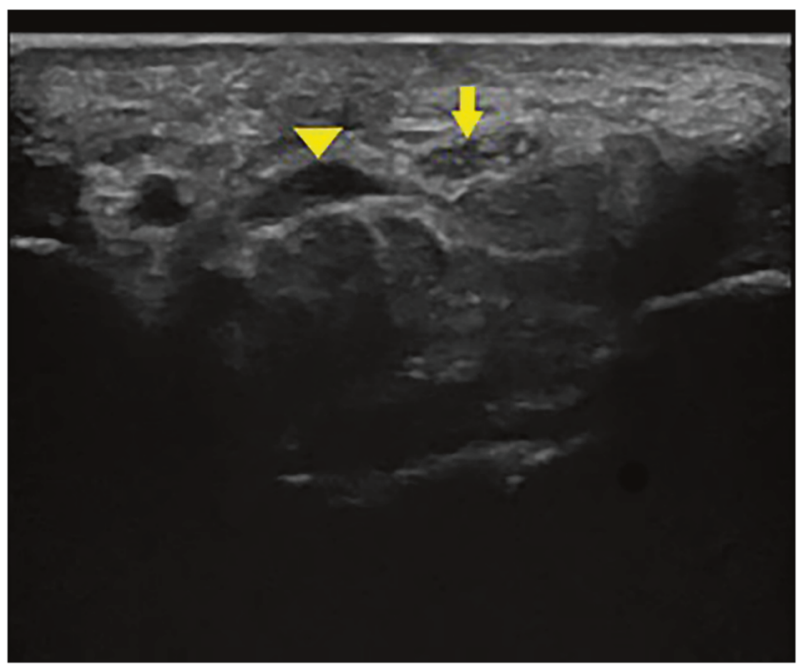

Fig. 1 Aberrant muscle belly of flexor digitorum superficialis of the index finger (arrowhead) is present next to median nerve (arrow)

basketball player presented to our electrodiagnostic laboratory with complaints of bilateral hand numbness after intensive training for a local veteran wheelchair basketball tournament. Previously, the patient had undergone carpal tunnel release but he reported that he did not gain complete relief from symptoms. Motor nerve conduction studies of the median nerve revealed evident prolonged distal latency. Right sensory nerve conduction study showed prolonged latency and delayed conduction velocity while left median sensory nerve action potential could not be elicited. The nerve conduction study findings were compatible to rightsided moderate and left-sided severe neurophysiological carpal tunnel syndrome. Ultrasonographic assessment of left carpal tunnel revealed the presence of abnormal muscle in the carpal tunnel (Fig. 1). The hypoechoic muscle belly next to the median nerve was detected on a static image of the carpal tunnel on the transvers plane at the pisiform bone level. The muscle belly was traced to the forearm, and it was identified as the flexor digitorum. Dynamic imaging was used to identify the muscle. While taking dynamic images of the carpal tunnel at the pisiform level, the patient's fingers were flexed, separately. Dynamic entrapment of the median nerve with the flexion of the index finger was viewed (see the Supplementary material). The patient was advised for rest and splinting and was referred to surgeon for revision surgery.

\section{Discussion}

Carpal tunnel syndrome is an important cause of wrist and hand pain and functional impairment in the general population, and also in individuals with spinal cord injury who use a wheelchair. It has been demonstrated that $49-73 \%$ of individuals with spinal cord injury who use manual wheelchairs have carpal tunnel syndrome [5]. Older age, female gender, increased weight, and greater body mass index (BMI) are found to be risk factors for carpal tunnel syndrome [5]. Besides these risk factors, repetitive weightbearing and mobility activities, which are specific to wheelchair use contribute to increased risk of development of carpal tunnel syndrome [5]. Previously, in two studies the carpal tunnel was evaluated ultrasonographically. Kim et al. [6] investigated the contributing factors of carpal tunnel syndrome electrodiagnostic and ultrasonographic findings of the median nerve. They found that median nerve swelling increased after wheelchair propulsion exercise. However, Abe et al. [7] did not find differences between persons with and without a disability in terms of the cross-sectional area of median nerve ultrasonographic measurements. However, both of the studies concentrated on median nerve crosssectional area measurements rather than assessment of anatomical structures within the tunnel. Aberrant muscles have been reported to be a cause of recurrence after initial classical carpal tunnel release $[1,8]$. The individual in this case had also undergone surgery and had persistent symptoms. Another causative factor for development of carpal tunnel syndrome in this case is the possible hypertrophy of the flexor digitorum muscle hypertrophy and intrusion due to intensive repetitive movements of the wrist. Cartwright et al. [9] investigated if muscle protrusion is a possible cause of carpal tunnel syndrome. They included 513 manual laborers and investigated the relationship between muscle protrusion and carpal tunnel syndrome. They found that muscle in carpal tunnel existed in all of the patients who were diagnosed with carpal tunnel syndrome while $90.9 \%$ of patients who were not diagnosed with carpal tunnel syndrome also had muscle intrusion. Their results support the cause of carpal tunnel syndrome in the present case. Individuals who repetitively use their hands may develop muscle protrusion into carpal tunnel due to hypertrophy resulting in carpal tunnel syndrome.

No definite curative treatment exists for carpal tunnel syndrome due to abberant muscles. Surgical treatments including open release of carpal tunnel with partial synovectomy around the flexor tendons, simple release of the muscle belly, debulking of muscle belly with maintaining integrity of the tendon itself were attempted. Entrapment symptoms disappeared immediately after the surgery and also there had been no recurrence of the symptoms during follow-up $[1,8,10]$.

A thorough medical history, physical examination, and electrodiagnostic studies are helpful for diagnosing carpal tunnel syndrome. Additionally, ultrasonography enables rapid imaging of the carpal tunnel anatomy and live visualization of dynamic changes within the carpal tunnel. Preservation of upper limb function is essential in 
individuals with spinal cord injury because it is crucial for functional independence. Therefore, it is important to determine the etiology of carpal tunnel syndrome. To the best of our knowledge, this is the first report of hypertrophy of the flexor digitorum muscle as a cause of carpal tunnel syndrome in a patient with spinal cord injury. Further studies investigating the prevalence and the role of aberrant muscles in the etiology of carpal tunnel syndrome in individuals with spinal cord injury should be conducted.

\section{Compliance with ethical standards}

Conflict of interest The authors declare that they have no conflict of interest.

Publisher's note: Springer Nature remains neutral with regard to jurisdictional claims in published maps and institutional affiliations.

\section{References}

1. Poelaert F, Van Geluwe F, Van Holder C. Persisting complaints after carpal tunnel release: nerve compression by the palmaris profundus muscle. J Hand Surg (Asian-Pac Vol). 2018;23:593-5.

2. Shimizu A, Ikeda M, Kobayashi Y, Saito I, Mochida J. Carpal tunnel syndrome with wrist trigger caused by hypertrophied lumbrical muscle and tenosynovitis. Case Rep Orthop. 2015;2015:705237. https://doi.org/10.1155/2015/705237.

3. Landi A, Acciaro AL, Della Rosa N, Pellacani A. Carpal tunnel syndrome: rare causes. In: Luchetti R, Amadio P. (eds) Carpal tunnel syndrome. Springer; Berlin, Heidelberg, 2007.

4. Presazzi A, Bortolotto C, Zacchino M, Madonia L, Draghi F. Carpal tunnel: Normal anatomy, anatomical variants and ultrasound technique. J Ultrasound. 2011;14:40-6.

5. Yang J, Boninger ML, Leath JD, Fitzgerald SG, Dyson-Hudson TA, Chang MW. Carpal tunnel syndrome in manual wheelchair users with spinal cord injury: a cross-sectional multicenter study. Am J Phys Med Rehabil. 2009;88:1007-16.

6. Kim DK, Kim BS, Kim MJ, Kim KH, Park BK, Kim DH. Electrophysiologic and ultrasonographic assessment of carpal tunnel syndrome in wheelchair basketball athletes. Ann Rehabil Med. 2017;41:58-65.

7. Abe M. Ultrasound measurement of the median nerve for carpal tunnel syndrome of the non-handicapped and the handicapped. J Phys Ther Sci. 2004;16:107-14.

8. Castillo R, Sheth K, Babigian A, Scola C. Recurrent carpal tunnel syndrome associated with extension of flexor digitorum muscle bellies into the carpal tunnel: A case series. Arch Plast Surg. 2018;45:474-8.

9. Cartwright MS, Walker FO, Newman JC, Arcury TA, Mora DC, Haiying C, et al. Muscle intrusion as a potential cause of carpal tunnel syndrome. Muscle Nerve. 2014;50:517-22.

10. Shimizu A, Ikeda M, Kobayashi Y, Saito I, Mochida J. Carpal tunnel syndrome with wrist trigger caused by hypertrophied lumbrical muscle and tenosynovitis. Case Rep Orthop. 2015;2015:705237. 